\title{
EQUIVALENT CONDITIONS FOR THE TIGHTNESS OF A SEQUENCE OF CONTINUOUS HILBERT VALUED MARTINGALES
}

\author{
MICHEL MÉTIVIER AND SHINTARO NAKAO*
}

\section{Introduction}

In [1] D. Aldous gave a sufficient condition for the tightness of a sequence $\left(X^{n}\right)_{n \geq 0}$ of right continuous (with left limits) processes taking their values in a separable complete metric space $S$. As already noted by Aldous this condition is far from being necessary when the processes $X^{n}$ are not continuous. More precisely the Aldous-condition implies the left-quasicontinuity of all the weak limits of the sequence $\left(X^{n}\right)_{n \geq 0}$. (see [1] or [4]).

When the $X^{n}$ 's are real square integrable martingales (or more generally locally square integrable martingales), it has been shown by $R$. Rebolledo ([9, see also an exposition in [4]) that the Aldous-condition for the positive increasing Meyer-processes $\left(\left\langle X^{n}\right\rangle\right)$ implies the Aldous-condition for $\left(X^{n}\right)_{n \geq 0}$.

In the case of Hilbert valued martingales it has been shown in [6] that the Aldous-condition on $\left(\left\langle X^{n}\right\rangle\right)$ plus a tightness condition on the sequence $\left(\left\langle\left\langle X^{n}\right\rangle_{T}\right)_{n \geq 0}\right.$ of operator valued random variables, $\left\langle\left\langle X^{n}\right\rangle\right.$ being the "tensorMeyer-process" of $X^{n}$ (see [7]), is also sufficient for the tightness of $\left(X^{n}\right)_{n \geq 0}$.

But in general neither the Aldous-condition on $\left(\left\langle\left\langle X^{n}\right\rangle\right\rangle\right)_{n \geq 0}$ is necessary for the tightness of $\left(X^{n}\right)_{n \geq 0}$, nor the tightness of $\left(\left\langle X^{n}\right\rangle\right)_{n \geq 0}$ alone implies the tightness of $\left(X^{n}\right)_{n \geq 0}$ (see J. Jacod, J. Mémin, M. Métivier [3]) unless some condition is assumed on the limits of the laws of the processes $\left\langle\left\langle X^{n}\right\rangle\right.$. When the processes are real or finite dimensional, the fact that the limiting laws are carried by the subset of continuous paths in $D\left(\boldsymbol{R}_{+}, \boldsymbol{H}\right)$ is sufficient. (see R. Rebolledo [9] and also [3] Theorem 1).

Considering only continuous processes, S. Nakao ([8]) recently proved

Received December 17, 1985.

(*) This paper was done while the first author was visiting the Department of Mathematics of the University of Nagoya, with the support of the Japan Society for the Progress of Sciences. 
an analogous result for Hilbert valued martingales. He showed that the tightness of $\left(X^{n}\right)_{n \geq 0}$ and the tightness of the operator-valued processes $\left(\left\langle X^{n}\right\rangle\right)_{n \geq 0}$ are equivalent when the $X^{n}$ are continuous Hilbert valued martingales. His proof is "direct", without reference to the Aldous-condition.

In this paper we prove that in the continuous case the tightness of $\left(X^{n}\right)_{n \geq 0}$ actually implies the Aldous-condition for $\left(\left\langle X^{n}\right\rangle\right)_{n \geq 0}$ and the tightness of marginals of $\left(\left\langle\left\langle X^{n}\right\rangle_{T}\right)_{n \geq 0}\right.$. As a consequence of a result in [6] we get a set of equivalent conditions for tightness containing in particular S. Nakao's result.

\section{Definitions and statement of the theorem}

Let $\left(X^{n}\right)_{n \geq 0}$ be a sequence of processes with values in a separable complete metric space $S$ with distance $d$. We assume that each process $X^{n}$ is defined on a probability space $\left(\Omega^{n},\left(\mathscr{F}^{n}\right), P_{n}\right)$ with its own filtration $\left(\mathscr{F}_{t}^{n}\right)_{t \in[0, T]}$.

2.1. We say that the sequence $\left(X^{n}\right)_{n \geq 0}$ satisfies the Aldous condition, which, from now on, we designate by [A], if for any $\eta>0$ and $\varepsilon>0$ there exists $\delta>0$ such that for every $n$ and every $\left(\mathscr{F}_{t}^{n}\right)$-stopping time $\tau^{n}$ on $\Omega^{n}$

$$
\sup _{0 \leq \theta \leq \delta} P_{n}\left\{d\left(X_{\tau^{n}+\theta}^{n}, X_{\tau^{n}}^{n}\right)>\eta\right\} \leq \varepsilon .
$$

2.2. We say that for $t \in[0, T]$ the sequence satisfies the condition $\left[\mathrm{T}_{t}\right]$ if the sequence $\left(X_{t}^{n}\right)_{n \geq 0}$ of $S$-valued random variables is tight, i.e.: for every $\varepsilon>0$ there exists a compact $K_{\varepsilon}$ in $S$ such that:

$$
P_{n}\left\{X_{t}^{n} \notin K_{\varepsilon}\right\} \leq \varepsilon .
$$

Let us call $D(T, S)$ (resp. $C(T, S)$ ) the set of mappings from $[0, T]$ in $S$ which are right continuous and have left limits in every $t \in[0, T]$ (resp. which are continuous), endowed with the Skorokhod topology (see Billingsley [2]) (resp. with the topology of uniform convergence). We call $\tilde{P}_{n}$ the law of $X^{n}: \tilde{P}_{n}$ is the image of $P_{n}$ by the mapping $\omega \backsim X^{n}(\omega, \cdot)$. If $X^{n}$ is continuous, $\tilde{P}_{n}$ is carried by the closed subset $C(T, S)$ of $D(T, S)$.

D. Aldous proved that if $\left(X^{n}\right)_{n \geq 0}$ verifies the conditions [A] and [ $\left.\mathrm{T}_{t}\right]$ for a dense set of $t \in[0, T]$ then $\left(X^{n}\right)_{n \geq 0}$ is tight. The converse is not true (see [1]). However, when the processes $X^{n}$ are continuous one has the following easy lemma: 
2.3. Lemma 1. If the processes $\left(X^{n}\right)_{n \geq 0}$ are continuous and if their laws $\tilde{P}_{n}$ form a tight sequence in $C(T, S)$, then the conditions $[\mathrm{A}]$ and $\left[\mathrm{T}_{t}\right]$ for every $t \in[0, T]$ hold.

This lemma is an easy consequence of the Ascoli theorem on the characterization of compact sets in $C(T, S)$.

2.4. Definitions. We recall the following definitions and notations. $\boldsymbol{H}$ being a real Hilbert space (the dual of which will be identified with $\boldsymbol{H}$ itself as long as there is no possible confusion), with scalar product $(\cdot, \cdot)$, we denote by $\mathscr{L}_{\infty}(\boldsymbol{H}, \boldsymbol{H})$ (resp. $\mathscr{L}_{2}(\boldsymbol{H}, \boldsymbol{H})$, resp. $\left.\mathscr{L}_{1}(\boldsymbol{H}, \boldsymbol{H})\right)$ the vector space of bounded linear operators in $\boldsymbol{H}$ with the operator norm (resp. the Hilbert space of Hilbert-Schmidt operators with the Hilbert-Schmidt norm \|\|$_{2}$, resp. the Banach space of nuclear operators with the nuclear norm \|\|$_{1}$ ).

Let $M$ be an $\boldsymbol{H}$-valued right continuous square-integrable martingale. We denote by $\langle\langle M\rangle$ the unique (up to indistinguishability) predictable $\mathscr{L}_{1}(\boldsymbol{H}, \boldsymbol{H})$-valued process, with the following property: for every $f, g \in \boldsymbol{H}$ the process $Y^{f, g}$ defined by

$$
Y_{t}^{f, g}:=\left(M_{t}, f\right)\left(M_{t}, g\right)-\left(M_{0}, f\right)\left(M_{0}, g\right)-\left(f,\langle M\rangle_{t} g\right)
$$

is a martingale.

Actually $\langle M\rangle$ takes its values in $\mathscr{L}_{1}^{+, s}(H, H)$, the cone of positive symmetric nuclear operators.

Now we write

$$
\langle M\rangle:=\text { trace of }\langle\langle M\rangle .
$$

$\langle M\rangle$ is a predictable (continuous if $M$ is continuous) positive increasing process with the property that $\left(\left\|M_{t}\right\|^{2}-\left\|M_{0}\right\|^{2}-\langle M\rangle_{t}\right)_{t \geq 0}$ is a martingale.

These definitions are easily extended to locally square integrable martingales.

The result of this paper is the following:

2.5. Theorem. Let $\left(M^{n}\right)_{n \geq 0}$ be a sequence of $\boldsymbol{H}$-valued continuous local martingales. Then the following properties are equivalent:

a) The laws $\left(\tilde{P}_{n}\right)_{n \geq 0}$ of the processes $M^{n}$ form a tight sequence of probabilities on $C(T, H)$.

b) Conditions [A] and $\left[\mathrm{T}_{t}\right], t \in[0, T]$ hold for the sequence $\left(M^{n}\right)_{n \geq 0}$.

$\left.\mathrm{b}^{\prime}\right) \quad J$ being a dense subset of $[0, T]$, conditions $[\mathrm{A}]$ and $\left\{\left[\mathrm{T}_{t}\right]: t \in J\right\}$ hold for the sequence $\left(M^{n}\right)_{n \geq 0}$. 
$\left.c_{1}\right)$ The laws $\left(\tilde{Q}_{n}\right)_{n \geq 0}$ of the processes $\left.\left(\left\langle M^{n}\right\rangle\right\rangle^{1 / 2}\right)_{n \geq 0}$ form a tight sequence of probabilities on $C\left(T, \mathscr{L}_{2}^{+}(H, H)\right)$.

$\left.\mathrm{c}_{2}\right)$ The laws $\left(\tilde{Q}_{n}^{1}\right)_{n \geq 0}$ of the processes $\left(\left\langle M^{n}\right\rangle\right)_{n \geq 0}$ form a tight sequence of probabilities on $C\left(T, \mathscr{L}_{1}^{+}(H, H)\right)$.

$\left.\mathrm{d}_{1}\right) J$ being a dense subset of $[0, T]$, condition [A] holds for the sequence $\left(\left\langle M^{n}\right\rangle\right)_{n \geq 0}$ and $\left\{\left[\mathrm{T}_{t}\right]: t \in J\right\}$ holds for the sequence $\left.\left(\left\langle M^{n}\right\rangle\right\rangle^{1 / 2}\right)_{n \geq 0}$.

$\mathrm{d}_{2}$ ) $J$ being a dense subset of $[0, T]$, condition [A] holds for the sequence $\left(\left\langle M^{n}\right\rangle\right)_{n \geq 0}$ and condition $\left\{\left[\mathrm{T}_{t}\right]: t \in J\right\}$ holds for the sequence $\left(\left\langle M^{n}\right\rangle\right)_{n \geq 0}$.

\section{Proof of the Theorem}

Lemma 1 gives a) $\Rightarrow$ b). Since, for $s \leq t$

$$
\left\langle M^{n}\right\rangle_{t}-\left\langle M^{n}\right\rangle_{s}=\operatorname{trace}\left(\left\langle\left\langle M^{n}\right\rangle_{t}-\left\langle\left\langle M^{n}\right\rangle_{s}\right)=\|\left\langle M^{n}\right\rangle_{t}-\left\langle\left\langle M^{n}\right\rangle_{s} \|_{1}\right.\right.\right.
$$

Lemma 1 also gives $c_{2}$ ) $\Rightarrow d_{2}$ ).

The mapping $\Phi: u \rightarrow u \circ u$ from $\mathscr{L}_{2}^{+, s}(\boldsymbol{H}, \boldsymbol{H})$ into $\mathscr{L}_{1}^{+, s}(\boldsymbol{H}, \boldsymbol{H})$ being continuous, one to one and with continuous inverse (see appendix), the sequences $\left.\left(\left\langle M^{n}\right\rangle\right\rangle^{1 / 2}\right)_{n \geq 0}$ and $\left(\left\langle M^{n}\right\rangle\right)_{n \geq 0}$ are together tight or not. Therefore the following equivalences are trivial: $\left.\left.\left.d_{1}\right) \Leftrightarrow d_{2}\right), c_{1}\right) \Leftrightarrow c_{2}$ ). Since $\left.b\right) \Rightarrow b^{\prime}$ ) is also trivial that the implications $\left.b^{\prime}\right) \Rightarrow a$ ) and $\left.d_{2}\right) \Rightarrow c_{2}$ ) are proved in [1] and the implication $\left.d_{1}\right) \Rightarrow a$ ) is proved in [6], we have only to show: a) $\Rightarrow d_{1}$ ).

Let us set for any $\mathscr{F}_{t}^{n}$-stopping time $\tau_{n}$ :

$$
Y_{t}^{n, \tau_{n}}:=\sup _{\tau_{n} \leq s \leq \tau_{n}+t}\left\|M_{s}^{n}-M_{\tau_{n}}^{n}\right\|^{2}
$$

For every stopping time $\sigma$

$$
E\left(\left\langle M^{n}\right\rangle_{i_{n}+\sigma}-\left\langle M^{n}\right\rangle_{\tau_{n}}\right)=E\left(\left\|M_{\tau_{n}+\sigma}^{n}-M_{\tau_{n}}^{n}\right\|^{2}\right)
$$

We make use of the following particular case of a lemma due to Lenglart (see [5]).

LEMMA 2. Let $X$ be an adapted positive process on some stochastic basis $\left(\Omega, \mathscr{F},\left(\mathscr{F}_{t}\right)_{t \in R_{+}}, P\right)$ and $Y$ be a positive adapted increasing continuous process such that for every stopping time $\sigma$

$$
E\left(X_{\sigma}\right) \leq E\left(Y_{\sigma}\right) \text {. }
$$

Then, for every stopping time $\sigma$, every $\eta>0, a>0$

$$
P\left\{\sup _{s \leq \sigma} X_{s}>\eta\right\} \leq \frac{a}{\eta}+P\left\{Y_{\sigma} \geq a\right\}
$$


In view of (3.1) we apply this lemma with $X_{t}:=\left\langle M^{n}\right\rangle_{\tau_{n}+t}-\left\langle M^{n}\right\rangle_{\tau_{n}}$ and $Y_{t}:=Y_{t}^{n, \tau_{n}}$. We thus obtain for every $a$ and $\eta$

$$
\left.P_{n}\left\{\left\langle M^{n}\right\rangle_{\tau_{n}+\delta}-\left\langle M^{n}\right\rangle_{z_{n}}\right\rangle \eta\right\} \leq a / \eta+P_{n}\left\{Y_{\delta}^{n, \tau_{n}} \geq a\right\} .
$$

$\eta$ being fixed we choose $a$ such that $a / \eta \leq \varepsilon / 2$ and then, using the property [A] of $\left(M^{n}\right)_{n \geq 0}$ which holds as a consequence of Lemma 1, we can choose $\delta$ such that

$$
P_{n}\left\{Y_{\dot{o}}^{n, z_{n}} \geq a\right\} \leq \frac{\varepsilon}{2} \quad \text { for all } n .
$$

We have then proved the property [A] for the sequence $\left(\left\langle M^{n}\right\rangle\right)_{n \geq 0}$. Setting $\tau_{n}=0$ in the formula (3.3) we get

$$
P_{n}\left\{\left\langle M^{n}\right\rangle_{t}>\eta\right\} \leq a / \eta+P_{n}\left\{\sup _{0 \leq s \leq T}\left\|M_{s}^{n}\right\|^{2} \geq a\right\} .
$$

In order to prove that condition $\left[\mathrm{T}_{t}\right]$ is valid for the sequence $\left(\left\langle M^{n}\right\rangle_{t}^{1 / 2}\right)_{n \geq 0}$, it is enough to prove (see [6] Proposition 1.3) that, for every $\varepsilon>0, \eta>0$ there exists a finite dimensional subspace $G_{\varepsilon, \eta}$ of $\mathscr{L}_{2}(\boldsymbol{H}, \boldsymbol{H})$ such that, for all $n$

$$
\left.P_{n}\left\{\|\left\langle M^{n}\right\rangle\right\rangle_{t}^{1 / 2}-\prod_{G_{\varepsilon}, \eta}\left\langle\left\langle M^{n}\right\rangle\right\rangle_{t}^{1 / 2} \|_{2}>\eta\right\} \leq \varepsilon
$$

where $\prod_{G_{\varepsilon, \eta}}$ denotes the orthogonal projection on $G_{\varepsilon, \eta}$. But the tightness of $\left(M^{n}\right)_{n \geq 0}$ implies the existence of a finite dimensional subspace $\boldsymbol{H}_{\varepsilon, a}$ of $\boldsymbol{H}$ such that

$$
P_{n}\left\{\sup _{s \leq T}\left\|M_{s}^{n}-\prod_{\boldsymbol{H}_{\varepsilon, a}} M_{s}^{n}\right\| \geq a\right\} \leq \varepsilon / 2 .
$$

Observing that for every stopping time $\tau_{n}$

$$
E_{n}\left(\left\langle M^{n}-\prod_{\boldsymbol{H}_{\varepsilon, a}} M^{n}\right\rangle_{\tau_{n}}\right) \leq E_{n}\left(\sup _{s \leq \tau_{n}}\left\|M_{s}^{n}-\prod_{\boldsymbol{H}_{\varepsilon, a}} M_{s}^{n}\right\|^{2}\right)
$$

and using again the Lenglart-inequality, we obtain

$$
\begin{aligned}
P_{n}\left\{\left\langleM^{n}-\right.\right. & \left.\left.\prod_{\boldsymbol{H}_{\varepsilon, a}} M^{n}\right\rangle_{\tau_{n}}>\eta\right\} \\
& \leq a / \eta+P_{n}\left\{\sup _{s \leq \tau_{n}}\left\|M_{s}^{n}-\prod_{H_{\varepsilon, a}} M_{s}^{n}\right\|^{2} \geq a\right\} \leq a / \eta+\varepsilon / 2 .
\end{aligned}
$$

The finite dimensional subset $\boldsymbol{H}_{\varepsilon, a}$ of $\boldsymbol{H}$ can therefore be chosen in such a way that for all $t \leq T$

$$
P_{n}\left\{\left\langle M^{n}-\prod_{H_{\varepsilon}, a} M^{n}\right\rangle_{t}>\eta\right\} \leq \varepsilon,
$$

which can be read 


$$
\left.P_{n}\left\{\left\langle\prod_{H_{\varepsilon, a}^{1},} M^{n}\right\rangle_{t}\right\rangle \eta\right\} \leq \varepsilon .
$$

Let us note that the orthogonal decomposition $\boldsymbol{H}=\boldsymbol{H}_{\varepsilon, a}+\boldsymbol{H}_{\varepsilon, a}^{\perp}$ of $\boldsymbol{H}$ leads to an orthogonal decomposition of $\mathscr{L}_{2}(\boldsymbol{H}, \boldsymbol{H})$ which we write $\mathscr{L}_{2}(\boldsymbol{H}, \boldsymbol{H})$ $=\sum_{i, j=1}^{2} H_{i} \hat{\otimes}_{2} H_{j}$ with $\boldsymbol{H}_{1}:=\boldsymbol{H}_{\varepsilon, a}$ and $\boldsymbol{H}_{2}:=\boldsymbol{H}_{\varepsilon, a}^{\perp}$. Denoting by $\prod_{\boldsymbol{H}_{i} \hat{\otimes}_{2} \boldsymbol{H}_{j}}$ (resp. $\prod_{i}$ ) the orthogonal projection on $\boldsymbol{H}_{i} \hat{\otimes}_{2} \boldsymbol{H}_{j}$ in $\mathscr{L}_{2}(\boldsymbol{H}, \boldsymbol{H})$ (resp. on $\boldsymbol{H}_{i}$ in $\boldsymbol{H}$ ) one has the orthogonal decomposition in $\mathscr{L}_{\varepsilon}(\boldsymbol{H}, \boldsymbol{H})$ :

$$
\left\langle\left\langle M^{n}\right\rangle\right\rangle_{t}^{1 / 2}=\sum_{i, j=1}^{2} \prod_{i} \circ\left\langle\left\langle M^{n}\right\rangle_{t}^{1 / 2} \circ \prod_{j} .\right.
$$

But

$$
\| \prod_{i} \circ\left\langle\langle M ^ { n } \rangle _ { t } ^ { 1 / 2 } \circ \prod _ { j } \| _ { 2 } ^ { 2 } \leq \| \prod _ { i } \circ \left\langle\left\langle M^{n}\right\rangle_{t}^{1 / 2} \|_{2}^{2}=\operatorname{trace} \prod_{i} \circ\left\langle\left\langle M^{n}\right\rangle_{t} \circ \prod_{i}=\left\langle\prod_{i} M\right\rangle_{i} .\right.\right.\right.
$$

The inequality (3.7) then leads to

$$
\begin{aligned}
& P_{n}\left\{\| \prod_{i} \circ\left\langle\left\langle M^{n}\right\rangle_{t}^{1 / 2} \circ \prod_{H_{t}, a} \|_{2}^{2}\right\rangle \eta\right\} \leq \varepsilon \quad i=1,2 \\
& P_{n}\left\{\| \prod_{H_{\ell, a}^{\perp},} \circ\left\langle\left\langle M^{n}\right\rangle_{t}^{1 / 2} \circ \prod_{i} \|_{2}^{2}\right\rangle \eta\right\} \leq \varepsilon \quad i=1,2
\end{aligned}
$$

and according to the orthogonal decomposition (3.8) this gives

$$
P_{n}\left\{\left\|\left\langle M^{n}\right\rangle_{t}^{1 / 2}-\prod_{H_{\varepsilon, a} \widehat{\otimes}_{2} H_{\varepsilon, a}}\left\langle\left\langle M^{n}\right\rangle\right\rangle_{t}^{1 / 2}\right\|_{2}^{2}>\eta\right\} \leq 3 \varepsilon .
$$

This proves (3.5) with $G_{\varepsilon, \eta}=\boldsymbol{H}_{\varepsilon, a} \hat{\otimes}_{2} \boldsymbol{H}_{\varepsilon, a}$ and therefore the theorem.

\section{Appendix}

For the convenience of the reader we give here a proof of the continuity of the mapping $v \backsim v^{1 / 2}$ from $\mathscr{L}_{1}^{+, s}(\boldsymbol{H}, \boldsymbol{H})$, the set of positive symmetric nuclear operators on $\boldsymbol{H}$ (with the nuclear norm) into $\mathscr{L}_{2}^{+, s}(\boldsymbol{H}, \boldsymbol{H})$, the set of symmetric positive Hilbert-Schmidt operators with the HilbertSchmidt norm. To this effect we consider a sequence $u_{n}$ in $\mathscr{L}_{2}^{+, s}(\boldsymbol{H}, \boldsymbol{H})$ such that $\lim _{n \rightarrow \infty}\left\|u_{n} \circ u_{n}-u \circ u\right\|_{1}=0$. Since

$$
\left\|u_{n}\right\|_{2}^{2}=\left\|u_{n} \circ u_{n}\right\|_{1} \quad \text { and } \quad\|u\|_{2}^{2}=\|u \circ u\|_{1}
$$

the following holds:

$$
\lim _{n \rightarrow \infty}\left\|u_{n}\right\|_{2}^{2}=\|u\|_{2}^{2} .
$$

Therefore we have only to prove that $u_{n}$ converges weakly to $u$ in the Hilbert space $\mathscr{L}_{2}^{+, s}(\boldsymbol{H}, \boldsymbol{H})$. But, since $\sup _{n}\left\|u_{n}\right\|_{2}<\infty$, the sequence $\left(u_{n}\right)$ is weakly compact and has weak limits. We have only to show that if $u^{\prime}$ is any limit then $u^{\prime}=u$. 
By definition, for every $\varphi \in \mathscr{L}_{2}(\boldsymbol{H}, \boldsymbol{H})$

$$
\lim _{n \rightarrow \infty} \operatorname{trace}\left(\left(u^{\prime}-u_{n}\right) \circ \varphi\right)=0 \text {. }
$$

Therefore

$$
\begin{aligned}
& \limsup _{n \rightarrow \infty}\left|\operatorname{trace}\left(u_{n} \circ u_{n}-u^{\prime} \circ u^{\prime}\right) \circ \varphi\right| \\
& \quad \leq \limsup _{n \rightarrow \infty}\left[\left|\operatorname{trace} u_{n} \circ\left(u_{n}-u^{\prime}\right) \circ \varphi\right|+\left|\operatorname{trace}\left(u_{n}-u^{\prime}\right) \circ u^{\prime} \circ \varphi\right|\right] \\
& \quad \leq \limsup _{n \rightarrow \infty}\left[\sup _{n}\left\|u_{n}\right\|_{2}\left|\operatorname{trace}\left(u_{n}-u^{\prime}\right) \circ \varphi\right|+\left|\operatorname{trace}\left(u_{n}-u^{\prime}\right) \circ u^{\prime} \circ \varphi\right|\right] \\
& \quad=0 .
\end{aligned}
$$

This shows that $\left(u_{n} \circ u_{n}\right)_{n \geq 0}$ converges to $u^{\prime} \circ u^{\prime}$ weakly in $\mathscr{L}_{2}(\boldsymbol{H}, \boldsymbol{H})$. But, since $\left(u_{n} \circ u_{n}\right)_{n \geq 0}$ converges to $u \circ u$ in $\mathscr{L}_{1}(\boldsymbol{H}, \boldsymbol{H})$ and therefore in $\mathscr{L}_{2}(\boldsymbol{H}, \boldsymbol{H})$, one has $u^{\prime} \circ u^{\prime}=u \circ u$. The $u_{n}$ 's being symmetric positive the same is true for $u^{\prime}$. Then $u=u^{\prime}$. This finishes the proof of the convergence of the sequence $\left(\mathrm{u}_{n}\right)_{n \geq 0}$ to $u$ in $\mathscr{L}_{2}(\boldsymbol{H}, \boldsymbol{H})$.

\section{REFERENCES}

[1] D. Aldous, Stopping times and tightness, Ann. of Prob., 6, no. 2 (1978), 335-340.

[2] P. Billingsley, Convergence of probability measures, Wiley, New York, 1968.

[ 3 ] J. Jacod, J. Mémin and M. Métivier, On tightness and stopping times, Stochastic Process. Appl., 14 (1983), 109-146.

[4] A. Joffe and M. Métivier, Weak convergence of sequences of semimartingales with applications to multitype branching processes, Advances in Applied Probability, 18 (1986), 20-65.

[ 5 ] E. Lenglart, Relation de domination entre deux processus, Ann. Inst. H. Poincaré, 13, no. 2 (1977), 171-179.

[6] M. Métivier, Convergence faible et principe d'invariance pour des martingales à valeurs dans des espaces de Sobolev, Ann. Inst. H. Poincaré, 20, no. 4 (1984), $329-348$.

[ 7 ] — Semimartingales, De Gruyter, Berlin-New York, 1982.

[8] S. Nakao, On weak convergence of sequences of continuous local martingales, Ann. Inst. H. Poincaré, 22, no. 3 (1986), 371-380.

[ 9 ] R. Rebolledo, La méthode des martingales appliquée à l'étude de la convergence en loi de processus, Mémoire de la S.M.F., t.62 (1979).

M. Métivier

Ecole Polytechnique

Palaiseau, France

S. Nakao

Department of Mathematics

Osaka University

Toyonaka, 560 Japan 